\title{
Effect of hiatal hernia size and columnar segment length on the success of radiofrequency ablation for Barrett's esophagus: A single-center, phase II clinical trial
}

\author{
Robert J. Korst, MD, ${ }^{\text {a,b }}$ Sobeida Santana-Joseph, MSN,,${ }^{\text {a,b }}$ John R. Rutledge, MAS, a Arthur Antler, MD, ${ }^{c}$ \\ Vivian Bethala, MD,${ }^{c}$ Anthony DeLillo, MD, ${ }^{c}$ Donald Kutner, DO, ${ }^{c}$ Benjamin E. Lee, MD,,${ }^{a, b}$ \\ Haleh Pazwash, MD, ${ }^{\mathrm{c}}$ Robert H. Pittman, MD, ${ }^{\mathrm{c}}$ Michael Rahmin, MD, ${ }^{\mathrm{c}}$ and Mitchell Rubinoff, $\mathrm{MD}^{\mathrm{c}}$
}

\begin{abstract}
Objective: Hiatal hernia is common in patients with Barrett's esophagus. We sought to evaluate the effect of hiatal hernia size and initial columnar segment length on the success of radiofrequency ablation of Barrett's esophagus.
\end{abstract}

\begin{abstract}
Methods: A phase II clinical trial was conducted aimed at evaluating the success of radiofrequency ablation in eradicating Barrett's esophagus. Success was defined as complete replacement of the columnar lining with squamous mucosa and lack of intestinal metaplasia using light microscopy. Hiatal hernia size and columnar segment length were measured endoscopically.

Results: Sixty-seven patients were accrued to the protocol. In the 55 patients who completed radiofrequency ablation (43 successes, 12 failures), the mean hiatal hernia size was $3.3 \mathrm{~cm}$ (range, $0-10 \mathrm{~cm}$ ), and the mean columnar segment length was $5.4 \mathrm{~cm}$ (range, 1-18 cm). The median length of the columnar segment was $3 \mathrm{~cm}$ in the successful cases and $8.5 \mathrm{~cm}$ in the failed cases $(P=.002)$. Although the median hiatal hernia size was identical in the successful and failed cases $(3 \mathrm{~cm}, P=.38)$, the median hiatal hernia size was $7 \mathrm{~cm}(P=.001)$ in the 6 patients who experienced nonhealing after the initial ablation. Patients who were successfully ablated but had larger hiatal hernias and longer columnar segment lengths required significantly more radiofrequency ablation sessions than those with smaller hernias and shorter segments $(P=.003$ and $P=.007$, respectively).
\end{abstract}

Conclusions: Patients with larger hiatal hernias and longer columnar segments are more likely to experience failure or nonhealing after radiofrequency ablation. These patients also require more radiofrequency ablation treatments to achieve successful eradication of Barrett's esophagus. (J Thorac Cardiovasc Surg 2011;142:1168-73)

Barrett's esophagus (BE) is a premalignant condition of the esophagus characterized by columnar metaplasia. Patients with $\mathrm{BE}$ are thought to have an increased risk of developing esophageal adenocarcinoma when compared with the general population. ${ }^{1}$ Radiofrequency ablation (RFA) is a technology that has been developed to eradicate BE, replacing it with normal, stratified, squamous epithelium. Published literature reports that the success rate of RFA in eradicating $\mathrm{BE}$ ranges from approximately $45 \%$ to $100 \%^{2-12}$; however, characteristics of challenging and failed cases using RFA have not been clearly delineated. In addition, there remains a paucity of formal prospective clinical trials

From The Daniel and Gloria Blumenthal Cancer Center, ${ }^{a}$ Paramus, NJ; the Division of Thoracic Surgery, ${ }^{\mathrm{b}}$ Department of Surgery, and the Division of Gastroenterology, ${ }^{c}$ Department of Medicine, The Valley Hospital/Valley Health System, Ridgewood, NJ.

Disclosures: Authors have nothing to disclose with regard to commercial support. Received for publication June 8, 2011; revisions received June 29, 2011; accepted for publication July 19, 2011; available ahead of print Aug 16, 2011.

Address for reprints: Robert J. Korst, MD, The Daniel and Gloria Blumenthal Cancer Center, Director, Thoracic Surgery, The Valley Hospital/Valley Health System, 1 Valley Health Plaza, Paramus, NJ 07652 (E-mail: korsro@valleyhealth.com).

$0022-5223 / \$ 36.00$

Copyright (C) 2011 by The American Association for Thoracic Surgery doi:10.1016/j.jtcvs.2011.07.037 using this technology in the published literature. We conducted a phase II, prospective, single institution, pilot clinical trial aimed at evaluating the success of RFA in eradicating $\mathrm{BE}$, with emphasis placed on the impact of hiatal hernia size and initial columnar segment length on the likelihood of a successful outcome.

\section{MATERIALS AND METHODS \\ Clinical Trial Design}

A single-arm, phase II trial of RFA for BE was conducted at a single institution after approval by the institutional review board. All patients with $\mathrm{BE}$ were eligible, regardless of the presence or absence of dysplasia, and all signed informed consent. BE was defined as an esophageal columnar segment greater than $3 \mathrm{~cm}$ in length regardless of whether or not intestinal metaplasia (IM) was detected by biopsy or an esophageal columnar segment less than $3 \mathrm{~cm}$ with biopsy-proven IM. Patients with a squamous lined tubular esophagus, with biopsies of the gastroesophageal junction showing only IM (termed "IM of the gastroesophageal junction") were not eligible, as were those who could not tolerate proton pump inhibitors. Patients who underwent prior fundoplication were eligible.

All patients underwent standardized video endoscopic assessment of BE before enrollment, which included white light and narrow band imaging (NBI), as well as 4-quadrant biopsies performed at 1 - to 2 -cm intervals throughout the columnar segment using jumbo biopsy forceps (Radial jaw 4; Boston Scientific, Natick, Mass). Hiatal hernias were sized by endoscopically measuring the distance from the incisors to the top of the gastric folds and subtracting this figure from the corresponding measurement of 


\section{Abbreviations and Acronyms \\ $\mathrm{BE}=$ Barrett's esophagus \\ $\mathrm{IM}=$ intestinal metaplasia \\ $\mathrm{NBI}=$ narrow band imaging \\ $\mathrm{RFA}=$ radiofrequency ablation}

the position of the crural pinch. Patients with visible lesions in their columnar segment had these removed using endoscopic mucosal resection (Duette, Wilson-Cook, Bloomington, Ind) before ablation. Endoscopic ultrasonography was not performed. Patients with T1a carcinomas were still eligible for inclusion provided that the endoscopic mucosal resection margins were negative, and computed tomography of the chest and positron emission tomography were negative for metastatic disease. Patients with $\mathrm{T} 1 \mathrm{~b}$ or deeper carcinomas were not eligible for inclusion. A minimum of 2 months was allowed to pass between the endoscopic mucosal resection procedure (if performed) and the first ablation.

\begin{abstract}
Ablation Protocol
All ablations were performed using the HALO system (BARRX Medical Inc, Sunnyvale, Calif), following strict adherence to the manufacturer's instructions. Ablations were performed in the endoscopy suite under conscious sedation, unless it was deemed unsafe by the ablating physician or anesthesiologist. In these later cases, the ablation was performed in the operating room under general anesthesia using an endotracheal tube and mechanical ventilation. A single investigator (R.J.K.) performed or assisted in all ablation procedures. In general, columnar segments greater than $3 \mathrm{~cm}$ in length were initially ablated using the HALO 360 system, and shorter segments were ablated using the HALO 90 system, although this decision was ultimately left up to the physician performing the ablation. The HALO 90 device was used to ablate the remaining "islands" of BE left over after a circumferential treatment. At least 8 weeks were allowed to elapse after each ablation procedure before another ablation was performed. The time period needed to complete RFA (in months) was therefore equal to the number of required RFA sessions multiplied by 2 months. All patients had their proton pump inhibitor dose doubled during the ablation period, and those not initially taking proton pump inhibitors were placed on a twice daily regimen.

The number of ablation sessions was not limited in this protocol. A given patient's treatment was considered completed when one of the following criteria was met:
\end{abstract}

- Voluntary withdrawal from the protocol for any reason.

- Failure of ablation as determined by the principal investigator (RJK).

- Successful ablation. A patient was considered successfully ablated if the tubular esophagus was completely eradicated of columnar lining as assessed by white light and NBI, and all biopsies obtained from the tubular esophagus lacked IM.

For the intention-to-treat analysis, all patients who met criteria for completion and were not successful, as described above, were considered failures.

The performance of hiatal hernia repair and fundoplication was left up to the discretion of the principal investigator (R.J.K.). In general, surgical repair was offered to those patients with poor symptom control on proton pump inhibitors or those who exhibited nonhealing after an initial ablation attempt, regardless of hiatal hernia size or columnar segment length.

\section{Data Collection}

Data collected on initial entry into the protocol included patient demographics, length of BE, size of hiatal hernia, proton pump inhibitor dose, and dysplasia (or carcinoma) status. Data collected during the ablation period included the number of ablation sessions per patient, ablation device (HALO 360 vs 90), compliance with proton pump inhibitor therapy, and percent of columnar mucosa successfully ablated with each session.

\section{Statistical Analysis}

Pearson's correlation coefficient $(r)$ was used to quantify the relationship between hiatal hernia size and initial columnar segment length. The nonparametric Wilcoxon Mann-Whitney test was used to determine the significance of the differences between median values of hiatal hernia size/initial columnar segment length for RFA successes, failures, and nonhealing patients. The nonparametric Kruskal-Wallis test was used to determine the significance of the effect of hiatal hernia size/initial columnar segment length on the number of ablation sessions needed to achieve success. IBM SPSS Statistics software (version 19; SPSS Inc, Chicago, Ill) was used for all analyses.

\section{RESULTS}

\section{Patient Characteristics}

Sixty-seven patients signed informed consent and were accrued into this phase II trial. Forty-six patients were male $(69 \%)$, and the mean age was 59.3 years (range, 24-85 years). Figure 1 demonstrates the distribution of Barrett's segment length and hiatal hernia size of all 67 patients. Hiatal hernia size was not significantly correlated with the initial length of BE in these 67 patients $(r=.18, P=.15)$. Fortyeight patients $(72 \%)$ had no dysplasia, 5 patients had lowgrade dysplasia (7.5\%), 6 patients had high-grade dysplasia
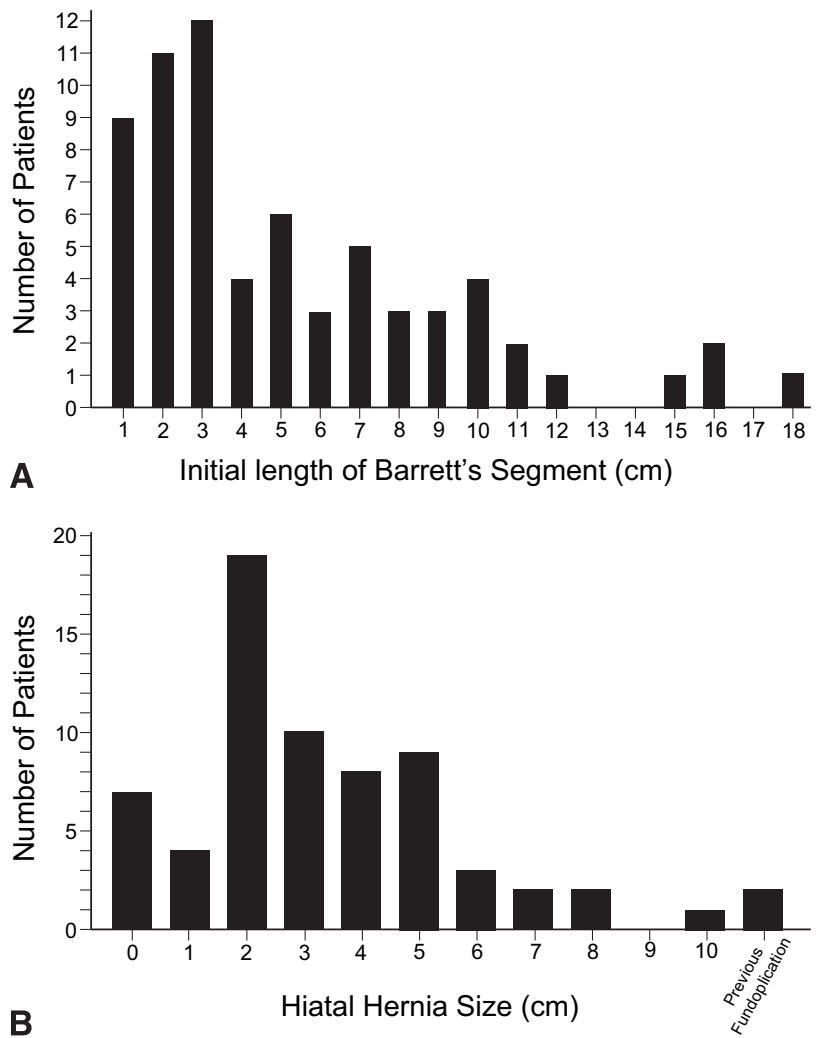

FIGURE 1. Distribution of initial BE length and hiatal hernia size in 67 enrolled patients. A, Initial BE length. B, Hiatal hernia size. 
(9\%), 1 patient had T1a adenocarcinoma (1.5\%), and 6 patients were indefinite for dysplasia $(9 \%)$. Fifty-five patients met the criteria for the completion of treatment as defined in the "Materials and Methods," and 12 patients are still actively being treated.

A total of 179 RFA treatment sessions were performed in these 67 patients. There were 54 HALO 360 procedures and 125 HALO 90 sessions. A total of 167 procedures were performed in the endoscopy suite under conscious sedation $(93 \%)$, and the remaining 12 procedures were performed in the operating room with patients under general anesthesia because of difficult airway management under conscious sedation. The initial ablation was performed using the HALO 360 device in 43 patients (64\%), and the HALO 90 device was used initially in 24 patients.

Six patients underwent hiatal hernia repair and fundoplication. In 3 patients, symptom control was poor before RFA initiation, resulting in surgical repair before ablation. In 3 others, surgical repair was performed after the first ablation attempt resulted in nonhealing, in an attempt to better control esophageal exposure to acid.

\section{Success of Radiofrequency Ablation in Eradicating Barrett's Esophagus}

According to the intention-to-treat principle, 43 of 55 patients who completed RFA had a successful result as defined in the "Materials and Methods," yielding an overall success rate of $78 \%$. Figure 2 shows the effect of initial BE length and hiatal hernia size on the success of RFA. The median initial BE lengths were significantly different between the

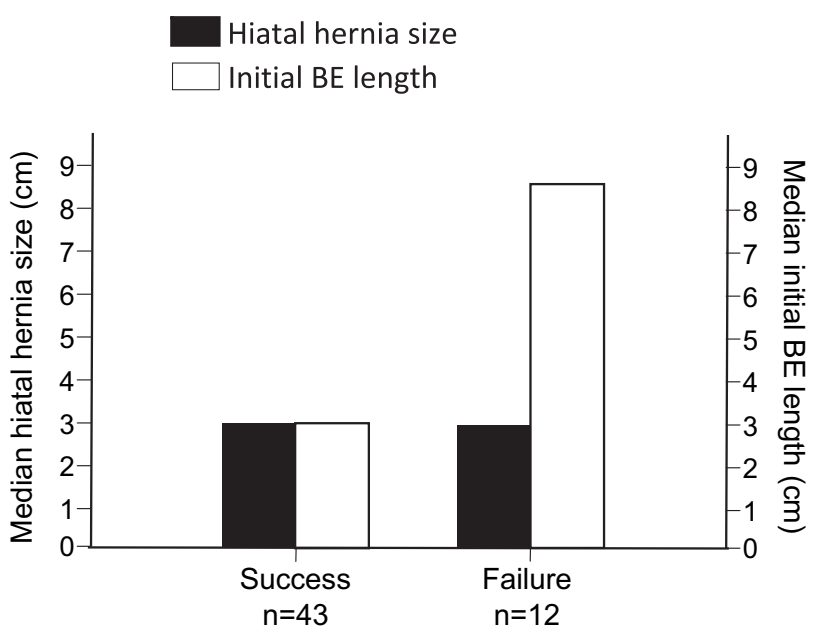

FIGURE 2. Effect of hiatal hernia size and initial columnar segment length on the ability to successfully ablate BE in the 55 patients who completed therapy. Compared with successfully ablated patients, patients who failed ablation had significantly longer initial BE segments $(8.5$ vs $3 \mathrm{~cm}$; $P=.002)$. No significant difference was seen between the successes and failures according to median hiatal hernia size $(P=.38)$ when analyzed by intention to treat. $B E$, Barrett's esophagus.
TABLE 1. Causes of failure of radiofrequency ablation for Barrett's esophagus

\begin{tabular}{lc}
\hline \multicolumn{1}{c}{ Cause } & No. of patients \\
\hline Insurance coverage denial & 3 \\
Other medical issues & \\
$\quad$ Progression of cardiac disease & 1 \\
Development of breast cancer & 1 \\
Motor vehicle accident & 1 \\
Voluntarily withdrew from protocol & 2 \\
Repeated nonhealing after ablation & 3 \\
Progression* & 1 \\
*Patient with multifocal high-grade dysplasia in whom raised lesions developed ne- \\
cessitating esophagectomy.
\end{tabular}

successes and failures $(P=.002)$, but this difference was not appreciated when hernia size was evaluated $(P=.38)$. As demonstrated in Table 1, 8 of 12 failures resulted from the inability to continue with the protocol for reasons not directly attributable to RFA itself. Only 4 patients demonstrated true failure of the technology, manifested by progression of disease or failure to heal appropriately after circumferential ablation, giving a "per protocol" success rate of 43 of 47 patients $(91 \%)$.

For the 43 patients successfully ablated, the median number of ablations necessary to achieve success was 2 (range, 1-8). Figure 3 demonstrates the effect of initial Barrett's segment length and hiatal hernia size on the number of ablation sessions needed to achieve success in these patients. Patients with longer columnar segment lengths and larger hiatal hernias required more RFA treatment sessions than those with shorter segments and smaller hernias $(P=.007$ and .003 , respectively).

\section{Nonhealing of the Ablated Segment}

Of the 55 initial ablations performed in the successfully ablated patients, $6(11 \%)$ resulted in nonhealing of the ablated segment 2 months later despite compliance with doubling of proton pump inhibitor dose and frequency as recommended by the manufacturer. Nonhealing was characterized endoscopically by replacement of the ablated area with an eschar, with no regeneration of mucosa. After 2 more months of follow-up, all patients were noted to have healed with recurrent columnar lining in the ablated segment. The median initial BE length in these patients was $8.5 \mathrm{~cm}$ (range, $5-11 \mathrm{~cm}$ ), and the median hiatal hernia size was $7 \mathrm{~cm}$ (range, $3-10 \mathrm{~cm}$ ). Table 2 demonstrates the clinical features and outcome of these 6 patients, and Figure 4 shows the effect of initial BE length and hiatal hernia size on the occurrence of nonhealing. The median initial $\mathrm{BE}$ lengths and hiatal hernia sizes were significantly different between the patients who were able to heal their mucosa and the nonhealing patients $(P=.015$ and .001 , respectively). All cases of nonhealing occurred after ablation with the HALO 360 device. 


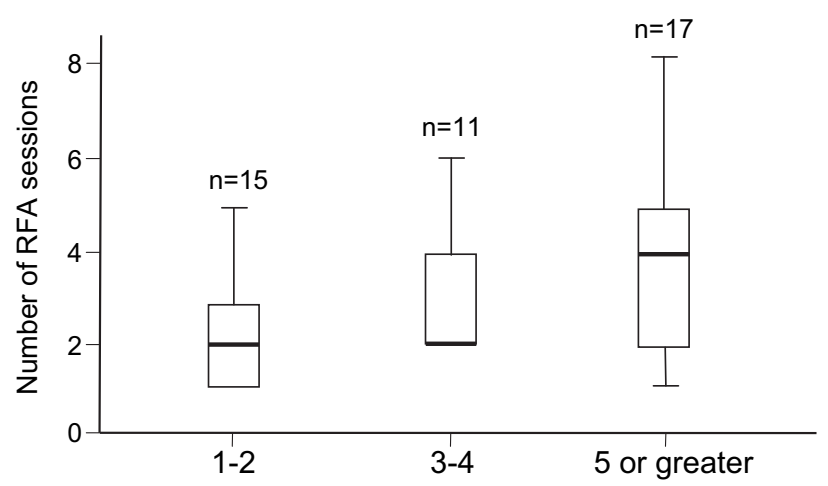

A Initial length of Barrett's Esophagus (cm)

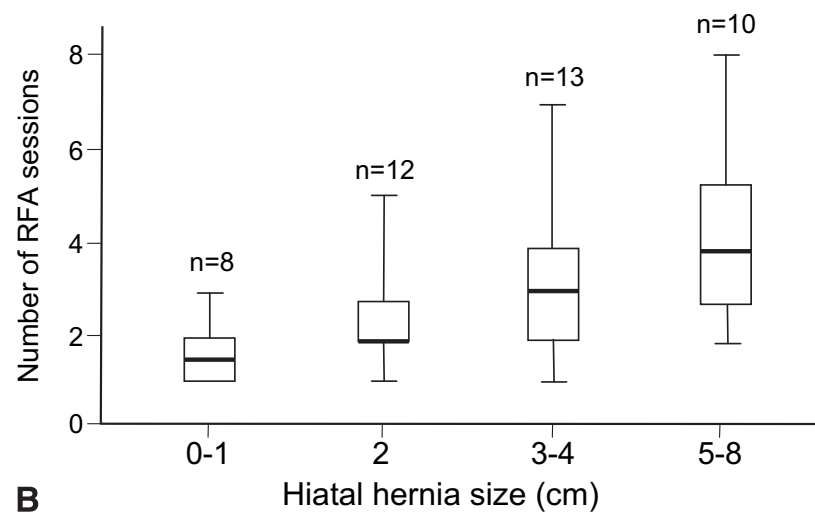

FIGURE 3. Effect of initial BE length and hiatal hernia size on the number of RFA sessions necessary for BE eradication in the 43 successfully ablated patients. A, Box and whisker plot of the number of RFA sessions needed to successfully eradicate BE according to the initial length of the columnar segment $(P=.007)$. The upper and lower ends of each box represent the upper and lower quartiles, the thick horizontal bar represents the median, and the extreme horizontal bars represent the maximum and minimum values. B, Box and whisker plot of the number of RFA sessions needed to successfully eradicate BE according to hiatal hernia size $(P=.003)$. The upper and lower ends of each box represent the upper and lower quartiles, the thick horizontal bar represents the median, and the extreme horizontal bars represent the maximum and minimum values. $R F A$, Radiofrequency ablation.

\section{DISCUSSION}

RFA for Barrett's esophagus is a relatively new technology that possesses the advantage of enhanced precision over previous ablative approaches. As a result, efficacy seems to be maximized, and adverse events are minimized compared with other ablation techniques, such as photodynamic therapy. ${ }^{13}$ Although many published reports regarding the use of RFA for BE exist, only a small number of these are prospective clinical trials. ${ }^{2-8}$ Formal prospective clinical trials provide the most robust data compared with retrospective and prospective case series because of their scientific rigor. With this as a background, we designed and performed a phase II, single-arm trial of RFA for BE.

\section{Success of Radiofrequency Ablation in Eradicating Barrett's Esophagus}

Current literature indicates that the success of RFA in the eradication of $\mathrm{BE}$ ranges from approximately $45 \%$ to $100 \% .^{2-12}$ In the present study, the success of RFA in eradicating $\mathrm{BE}$ was $78 \%$ when analyzed according to the intention to treat, which mirrors the success rate published in the largest RFA trial to date. ${ }^{2}$ One explanation for the wide range of success rates in the published literature is variation in ablation protocols, especially with regard to a predetermined maximum number of ablations. In the present trial, no predetermined limit was set on the maximum number of ablation sessions to determine the actual number of ablations necessary to achieve success in any given patient.

Another factor that may influence the reported success rates of RFA is the variable use of NBI after ablation. Many of the previously published trials evaluating RFA did not mandate the use of NBI or mention its use in the corresponding publication. ${ }^{2,3,6,7}$ In the present study, the protocol mandated the use of NBI, where it was not unusual for NBI to detect very small islands of columnar mucosa that went undetected under white light. In these cases, patients were re-ablated, using NBI as a guide.

\section{Impact of Hiatal Hernia Size and Columnar Segment Length on the Success of Radiofrequency Ablation}

In the present clinical trial, the length of the initial columnar segment affected both the ability to perform a successful ablation and the number of ablations needed to achieve success. This finding has also been alluded to in other published studies. ${ }^{2,14,15}$ Although the increased surface area of the columnar mucosa in the patients with longer segments

TABLE 2. Clinical features and outcome in 6 patients with nonhealing after initial ablation

\begin{tabular}{lccccc}
\hline Patient no. & Age $(\mathbf{y}) / \mathbf{g e n d e r}$ & Initial BE length $(\mathbf{c m})$ & Hiatal hernia size $(\mathbf{c m})$ & Further interventions & Outcome \\
\hline 1 & $47 / \mathrm{M}$ & 10 & 3 & None & Withdrew from trial* \\
2 & $53 / \mathrm{F}$ & 9 & 7 & None & Withdrew from trial $\dagger$ \\
3 & $72 / \mathrm{M}$ & 11 & 6 & Re-ablation $\ddagger$ & Failure $\|$ \\
4 & 8 & 7 & Re-ablation $\S$ & Failure $\|$ \\
5 & $54 / \mathrm{M}$ & 7 & 10 & Re-ablation $\ddagger$ & Failure $\|$ \\
6 & $67 / \mathrm{M}$ & 5 & 8 & Re-ablation $\ddagger$ & Success \\
\hline
\end{tabular}

*Moved out of area. †ंDeveloped metastatic breast cancer. †̦Further increased proton pump inhibition and performed hiatal hernia repair. §̧Further increased proton pump inhibition. ||Under surveillance using endoscopy and biopsies. 


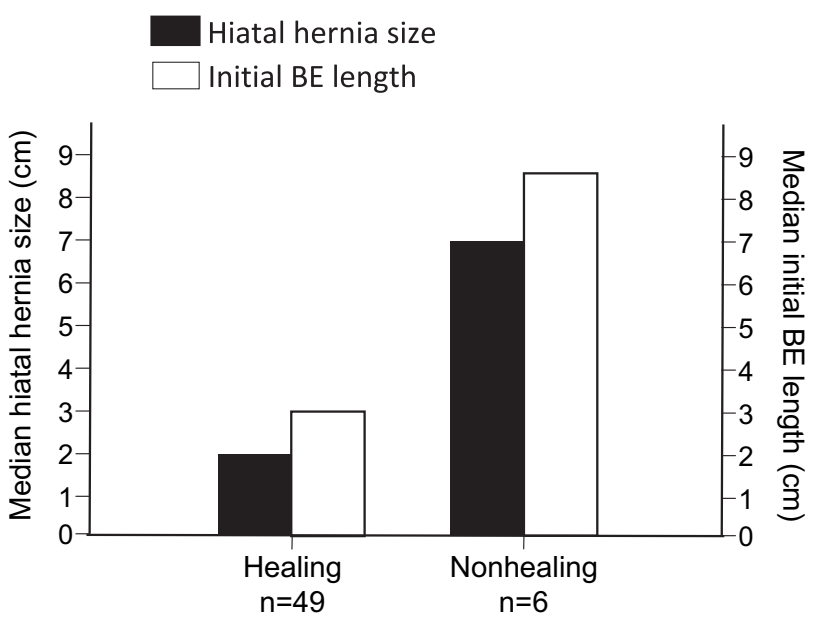

FIGURE 4. Effect of hiatal hernia size and initial columnar segment length on the phenomenon of nonhealing after RFA for BE in the 55 patients who completed therapy. Patients in whom the initial ablation resulted in nonhealing had significantly longer BE segments ( $8.5 \mathrm{vs} 3 \mathrm{~cm} ; P=.015)$ and significantly larger hiatal hernias ( $7 \mathrm{vs} 2 \mathrm{~cm} ; P=.001$ ) than those who were able to heal their ablated segment after the initial RFA session. $B E$, Barrett's esophagus.

may be responsible for this observation, another possible explanation may be that patients with longer columnar segments may have more severe reflux or an inherent propensity to form BE. The fact that the patients with longer segments also were more likely to experience the phenomenon of nonhealing after their initial ablation supports the latter of the 2 explanations.

Similar to initial columnar segment length, hiatal hernia size also affected the ability to successfully ablate BE. Although the ability to achieve success (Figure 2) did not seem to be affected, hiatal hernia size clearly seemed to affect the likelihood of nonhealing after initial ablation (Figure 4), as well as the number of ablations needed to achieve success (Figure 3). To our knowledge, this has not been reported. A possible explanation for this finding may be that larger, sliding hernias present an anatomic challenge for the HALO system, inasmuch as the esophagus may be somewhat tortuous in its course and the gastroesophageal junction may move significantly up and down. As with the initial columnar segment length, a larger hiatal hernia may also simply be a marker for more severe reflux disease, which may in turn affect success rates of RFA. For patients with large, severely symptomatic hiatal hernias and long, dysplastic columnar segments, esophagectomy may be a viable option. Modern, minimally invasive operative approaches may be particularly suitable for this population of patients.

\section{Nonhealing of the Esophagus After Radiofrequency Ablation}

Nonhealing, as defined in the "Results," represented the major reason for the technical failure of the HALO system in the present clinical trial. Patients with both long columnar segment lengths and large hiatal hernia sizes seemed to be predisposed to failure in this fashion. The phenomenon of nonhealing is not well described in the published literature. A recent publication by Herrero and colleagues ${ }^{16}$ described a series of patients with columnar segments greater than $10 \mathrm{~cm}$ and found similar nonhealing in 4 of 26 patients $(15 \%)$. Pouw and colleagues ${ }^{3}$ also reported a single patient (of 23) with a long columnar segment that "did not regenerate squamous mucosa" after ablation. ${ }^{3}$ In the present study, the frequency of nonhealing was 6 of 67 initial ablations $(9 \%)$.

The reason for nonhealing remains unknown. Herrero and colleagues ${ }^{16}$ hypothesized that these patients may have had poor reflux control. In the present trial, attempts were made to further control reflux in 4 of the 6 nonhealing patients, which included both increasing proton pump inhibitor dose and hiatal hernia repair and fundoplication. The fundoplication group underwent normal $\mathrm{pH}$ testing after surgery, but 2 of 3 patients still could not be successfully ablated (Table 2). This observation calls the hypothesis of poor reflux control into question.

\section{CONCLUSIONS}

We conducted a formal phase II clinical trial of RFA for $\mathrm{BE}$. This technology seems to eradicate BE in the majority of cases when analyzed according to the intention to treat. Problematic patients clearly seem to be those with large hiatal hernias and long columnar segments. Nonhealing represents a major cause of technical failure of this approach, for which further investigation is needed. Although the majority of patients in the present trial had nondysplastic BE, the role of RFA for patients without dysplasia outside of a clinical trial remains unclear and worthy of further study.

\section{References}

1. O'Connor JB, Falk GW, Richter JE. The incidence of adenocarcinoma and dysplasia in Barrett's esophagus: report on the Cleveland Clinic Barrett's Esophagus Registry. Am J Gastroenterol. 1999;94:2037-42.

2. Shaheen NJ, Sharma P, Overholt BF, Wolfsen HC, Sampliner RE, Wang KK, et al. Radiofrequency ablation in Barrett's esophagus with dysplasia. $N$ Engl J Med. 2009;360:2277-88.

3. Pouw RE, Wirths K, Eisendrath P, Sondermeijer CM, Ten Kate FJ, Fockens P, et al. Efficacy of radiofrequency ablation combined with endoscopic resection for Barrett's esophagus with early neoplasia. Clin Gastroenterol Hepatol. 2010;8:23-9.

4. Van Vilsteren FGI, Pouw RE, Seewald S, Herrero LA, Sondermeijer CMT, Visser M, et al. Stepwise radical endoscopic resection versus radiofrequency ablation for Barrett's Esophagus with high grade dysplasia or early cancer: a multicenter randomized trial. Gut. 2011;60:765-73.

5. Hernandez JC, Reicher S, Chung D, Pham BV, Tsai F, Disibio G, et al. Pilot series of radiofrequency ablation of Barrett's esophagus with or without neoplasia. Endoscopy. 2008;40:388-92.

6. Roorda AK, Marcus SN, Triadafilopoulos G. Early experience with radiofrequency energy ablation therapy for Barrett's esophagus with and without dysplasia. Dis Esophagus. 2007;20:516-22.

7. Sharma VK, Wang KK, Overholt BF, Lightdale CJ, Fennerty MB, Dean PJ, et al. Balloon-based, circumferential, endoscopic radiofrequency ablation of Barrett's esophagus: 1-year follow-up of 100 patients. Gastrointest Endosc. 2007;65: 185-95. 
8. Gundrie JJ, Pouw RE, Sondermeijer CM, Peters FP, Curvers WL, Rosmolen WD, et al. Stepwise circumferential and focal ablation of Barrett's esophagus with high-grade dysplasia: results of the first prospective series of 11 patients. Endoscopy. 2008;40:359-69.

9. Ganz RA, Overholt BF, Sharma VK, Fleischer DE, Shaheen NJ, Lightdale CJ, et al. Circumferential ablation of Barrett's esophagus that contains high-grade dysplasia: a U.S. multicenter registry. Gastrointest Endosc. 2008;68:35-40.

10. Velanovich V. Endoscopic endoluminal radiofrequency ablation of Barrett's esophagus: initial results and lessons learned. Surg Endosc. 2009;23: 2175-80.

11. Lyday WD, Corbett FS, Kuperman DA, Kalvaria I, Mavrelis PG, Shughoury AB, et al. Radiofrequency ablation of Barrett's esophagus: outcomes of 429 patients from a multicenter community practice registry. Endoscopy. 2010;42:272-8.
12. Eldaif SM, Lin E, Singh KA, Force SD, Miller DL. Radiofrequency ablation of Barrett's esophagus: short-term results. Ann Thorac Surg. 2009;87:405-10.

13. Fleischer DE, Sharma VK. Endoscopic ablation of Barrett's esophagus using the HALO system. Dig Dis. 2008;26:280-4.

14. O'Connell K, Velanovich V. Effects of Nissen fundoplication on endoscopic endoluminal radiofrequency ablation of Barrett's esophagus. Surg Endosc. 2011; 25:830-4.

15. Zehetner J, DeMeester SR, Hagen JA, Ayazi S, Augustin F, Lipham JC, et al. Endoscopic resection and ablation versus esophagectomy for high-grade dysplasia and intramucosal adenocarcinoma. J Thorac Cardiovasc Surg. 2011;141:39-47.

16. Herrero LA, van Vilsteren FGI, Pouw RE, ten Kate FJW, Visser M Seldenrijk CA, et al. Endoscopic radiofrequency ablation combined with endoscopic resection for early neoplasia in Barrett's esophagus longer than $10 \mathrm{~cm}$. Gastrointest Endosc. 2011;73:682-90.

\title{
Radiofrequency ablation for nondysplastic Barrett's esophagus: Should we do it, because we can?
}

\author{
Nirmal Veeramachaneni, MD
}

The treatment of patients with Barrett's esophagus has been a topic of intense investigation for the last several decades. Although it is well recognized that Barrett's esophagus may develop into dysplasia and then progress to invasive esophageal cancer, initial studies comparing fundoplication surgery and the best medical management available demonstrated no difference in either regression of the Barrett's esophagus or diminution of cancer risk. ${ }^{1}$ More modern randomized, controlled trials that investigated laparoscopic fundoplication or long-term proton pump inhibitor use also did not demonstrate any regression of Barrett's esophagus with either strategy ${ }^{2}$ after 5 years of follow-up. In fact, the American Gastroenterological Association does not currently recommend routine surgery (fundoplication) to treat Barrett's esophagus. ${ }^{3}$ Strategies to eradicate Barrett's esophagus by means of radiofrequency ablation in patients with high-grade dysplasia, however, have

\footnotetext{
From the Division of Cardiothoracic Surgery, University of North Carolina-Chapel Hill, Chapel Hill, NC.

Disclosures: Author has nothing to disclose with regard to commercial support.

Received for publication July 31, 2011; accepted for publication Aug 25, 2011; available ahead of print Aug 16, 2011.

Address for reprints: Nirmal Veeramachaneni, MD, Thoracic Surgery, University of North Carolina-Chapel Hill, 3040 Burnett, Womack CB 7065, Chapel Hill, NC

27599 (E-mail: ; nirmalv@med.unc.edu).

J Thorac Cardiovasc Surg 2011;142:1173-4

$0022-5223 / \$ 36.00$

Copyright (c) 2011 by The American Association for Thoracic Surgery

doi:10.1016/j.jtcvs.2011.08.034
}

demonstrated efficacy in preventing the progression to esophageal cancer. ${ }^{4}$

In the accompanying article in this issue of the Journal, Korst and colleagues ${ }^{5}$ have extended the application of radiofrequency ablation technology in a phase 2 trial of patients with Barrett's esophagus without evidence of dysplasia ( $72 \%$ of patients enrolled). This study demonstrated efficacy in eradicating Barrett's esophagus in 78\% of treated patients. Patients with large hiatal hernias were found to be at risk for impaired healing of the esophageal lining after radiofrequency ablation. This study provides valuable information on the intensity of follow-up and treatment necessary to treat Barrett's esophagus with radiofrequency ablation technology. There is considerable variation with respect to the number of radiofrequency ablation treatments needed, with as many as 5 treatments needed for patients with less than $2 \mathrm{~cm}$ Barrett's esophagus and as many as 8 treatments needed for patients with greater than $5 \mathrm{~cm}$ of Barrett's esophagus. Treatments were done at 2-month intervals. Although the study patients have undergone careful endoscopic follow-up, the long-term efficacy of the strategy remains unclear. Korst and colleagues ${ }^{5}$ are unable to provide long-term follow-up data, and the rate of recurrent Barrett's esophagus is unknown. In addition, there is no assessment for potential buried islands of Barrett's esophagus. Furthermore, given the low rate of progression of nondysplastic Barrett's esophagus to cancer, the utility of the intervention, even if successful, is unclear. 